\title{
CARACTERIZAÇÃO FÍSICA E FÍSICO-QUÍMICA DE MORANGOS SAN ANDREAS PRODUZIDOS SOB SISTEMAS ORGÂNICO E CONVENCIONAL
}

\author{
Antônio Nunes dos Santos ${ }^{1}$, Bruno Emanuel Souza Coelho ${ }^{2 *}$, Laura Cardoso Galvão ${ }^{3}$, Gesiel \\ Silva Torres ${ }^{3}$, Acácio Figueiredo Neto ${ }^{4}$, Iuri Honório dos Santos ${ }^{5}$.
}

\begin{abstract}
${ }^{1}$ Estudante do curso Técnico em Fruticultura, Fruticultura, Centro de Excelência em Fruticultura - Senar/BA, Juazeiro/BA. E-mail: antonionunes@mpmontagens.com.br.

${ }^{2}$ Programa de Pós-graduação em Agronomia - Produção Vegetal, Produção Vegetal - Fisiológica Pós-Colheita de Frutas e Hortaliças, Universidade Federal do Vale do São Francisco, campus Ciências Agrárias, Petrolina/PE. E-mail: souza.coelho.18@gmail.com.

3 Graduando (a) em Engenharia Agronômica, Produção vegetal - Tecnologia Pós-Colheita de Frutas e Hortaliças, Universidade Federal do Vale do São Francisco campus Ciências Agrárias, Petrolina/PE E-mail: laahcardoso8@gmail.com; gesieltorres.re@gmail.com.

5 Professor Adjunto do Colegiado de Engenharia Agrícola e Ambiental, Engenharia de Processamento e Armazenamento de Produtos Agrícolas, Universidade Federal do Vale do São, campus Juazeiro/BA, e-mail: acacio.figueiredo@univasf.edu.br.

${ }^{2}$ Instrutor, Agricultura de Precisão, Centro de Excelência em Fruticultura - Senar/AR BA, Juazeiro/BA E-mail: iuri_honorio@hotmail.com.
\end{abstract}

Recebido: 23/02//2020; Aceito: 03/09/2020

RESUMO: O Brasil é o segundo maior produtor de morangos da América Latina, e a Bahia é o estado com maior destaque nacional em termos de produtividade. A produção brasileira de morangos é oriunda principalmente do sistema agrícola convencional, caracterizado pelo uso intensivo agrotóxicos, que além do potencial poluidor do ambiente e danoso ao meio ambiente traduz o mesmo assunto. Neste contexto, a agricultura orgânica destaca-se como um sistema alternativo que visam atender aos requisitos de sustentabilidade ambiental, econômica e social. Desta forma, o trabalho teve por objetivo avaliar o efeito dos sistemas de produção convencional e orgânico, quanto as características de qualidade de morangos 'San Andreas' produzidos em Morro do Chapéu-BA. Os frutos foram colhidos ao atingirem o ponto de maturação fisiológica em áreas de sistemas de produção convencional e orgânico, localizadas no município de Morro do Chapéu-BA. Os frutos foram avaliados quanto aos parâmetros de: massa fresca, comprimento, largura, firmeza, $\mathrm{pH}$, acidez, sólidos solúveis, ratio, vitamina $\mathrm{C} \mathrm{e}$ cor. Os dados experimentais foram submetidos ao teste de normalidade, e as médias foram comparadas através do teste de $\mathrm{t}(p>0,05)$. Os morangos produzidos no sistema de produção orgânica em Morro do Chapéu-BA apresentaram maior massa fresca, e comprimento e largura, resultando em frutos maiores. Foi observado menores valores de acidez, sólidos solúveis, e maiores valores de $\mathrm{pH}$ e teor de vitamina $\mathrm{C}$ nos frutos produzidos em sistema de produção orgânica. Não houve diferença estatística na firmeza da polpa, e coloração da epiderme dos frutos em função do sistema de cultivo.

Palavras-chave: Agrotóxico. Fragaria $\times$ ananassa Duch. Morangueiro. Qualidade. Vitamina C. 


\title{
PHYSICAL AND PHYSICAL AND CHEMICAL CHARACTERIZATION OF SAN ANDREAS STRAWBERRIES PRODUCED UNDER ORGANIC AND CONVENTIONAL SYSTEMS
}

\begin{abstract}
Brazil is the second largest strawberry producer in Latin America and the state of Bahia with the greatest national prominence in terms of execution. The Brazilian production of strawberries is mainly the conventional agricultural system, the more intensive use of pesticides, in addition to the potential pollutant of the environment and harmful to the translated environment or even subject. In this context, organic agriculture includes an alternative visualization system that meets the requirements of environmental, economic and social sustainability. In this way, the work aimed to evaluate the effect of conventional and organic production systems, regarding the quality characteristics of 'San Andreas' strawberries used in Morro do Chapéu-BA. The fruits were harvested when reaching the point of physiological maturation in areas of conventional and organic production systems, located in the municipality of Morro do Chapéu-BA. The fruits were relatively low in terms of the parameters of: fresh weight, length, width, firmness, $\mathrm{pH}$, acidity, soluble solids, ratio, vitamin $\mathrm{C}$ and color. The experimental data were subjected to the normality test and the media were compared using the $t$ test $(p>0.05)$. The strawberries used in the organic production system at Morro do Chapéu-BA were the ones with the greatest fresh weight, length and width, resulting in larger fruits. Lower values of acidity, soluble solids and higher values of $\mathrm{pH}$ and vitamin $\mathrm{C}$ content were observed in the fruits used in the organic production system. There was no statistical difference in the firmness of the pulp and in the color of the fruit species depending on the cultivation system.
\end{abstract}

Key words: Pesticide. Fragaria $\times$ ananassa Duch. Strawberry plant. Quality. Vitamin C.

\section{INTRODUÇÃO}

O morango (Fragaria $\times$ ananassa Duch) é um pseudofruto resultado do desenvolvimento conjunto do receptáculo floral de diversas flores. Possui sabor doce, e elevada aceitabilidade sensorial, além de alto valor biológico, contendo expressivos teores de vitaminas A, C, B2, B3, B6, potássio, fósforo, cálcio, magnésio, sódio, ferro, zinco, folato e potássio, e seu consumo pode auxiliar no tratamento de doenças crônicas como câncer e doenças cardíacas (ŠAMEC et al., 2016; UNITED STATES DEPARTMENT OF AGRICULTURE - USDA, 2018).

A cultura do morangueiro é produzida e apreciada nas mais variadas regiões do mundo, sendo a China, os Estados Unidos da América e a Espanha, os maiores produtores mundiais (FAOSTAT, 2017; DIEL et al., 2018; ANTUNES et al., 2018). O cultivo do morango é grande importância econômica, sendo uma fruta amplamente explorada tanto para consumo in natura, quanto para a elaboração de derivados (XU et al., 2014).

O Brasil é o segundo maior produtor de morangos da América Latina, com produção variando entre 30 a 60 t/ha, e participação de $1 \%$ das exportações no cenário mundial (SERVIÇO BRASILEIRO DE APOIO ÀS MICRO E PEQUENAS EMPRESAS - SEBRAE, 
2017; DIAS et al., 2015). A Bahia é o estado com maior destaque em termos de produtividade, estima-se uma média de 40 t/ha, visto que os estados de São Paulo, Minas Gerais, Rio Grande do Sul e Paraná, que são os maiores produtores em termos de área cultivada, apresentam produtividade média de 34, 32, 25 e 21 t/ha, respectivamente (SEBRAE, 2017; ANTUNES et al., 2018).

A principal região produtora de morangos da Bahia é a Chapada Diamantina, ocorrendo em grande parte na agricultura familiar, com destaque para os municípios de Barra da Estiva, Ibicoara, e principalmente, Morro do Chapéu, decorrente das condições climáticas da região (SEBRAE, 2017).

A variedade de morango 'San Andreas' possui pseudofrutos grandes e de bom formato, elevada firmeza de polpa, sabor acentuado, e alta cromaticidade, o que torna a fruta muito atraente perante os consumidores. É uma variedade resistente a doenças, precoce e com uma produção estável durante o seu ciclo produtivo, adapta-se muito bem a diversos climas e mercados (SHAW; LARSON, 2008; RUAN; LEE; YEOUNG, 2013).

A produção brasileira de morangos é oriunda principalmente do sistema agrícola convencional, caracterizado pelo uso intensivo de agrotóxicos, utilizados no controle fitossanitário de pragas e doenças (COSTA et al., 2015), que geram resíduos com alto potencial poluidor ao meio ambiente, além de ser um fator de alto risco a saúde humana (KOBI et al., 2018).

Por outro lado, evidencia-se o aumento do interesse e a conscientização dos consumidores em relação à necessidade de consumir alimentos livres de resíduos químicos (REGANOLD et al., 2010). Neste contexto, a agricultura orgânica foi proposta como um sistema agrícola alternativo, sendo que, as tecnologias adotadas neste sistema agrícola visam atender aos requisitos de sustentabilidade ambiental, econômica e social (REGANOLD; WACHTER 2016).

De acordo com Andrade et al. (2017), o sistema de cultivo de plantas, alinhado a variedade em cultivo, tipo de solo, e clima, entre outros fatores, podem interferir na qualidade dos frutos, principalmente nas propriedades físicas e químicas.

Nesse sentido, este trabalho foi desenvolvido com o objetivo de avaliar o efeito dos sistemas de produção convencional e orgânico, sobre a qualidade de morangos 'San Andreas' produzidos em Morro do Chapéu - BA.

\section{MATERIAL E MÉTODOS}

Os frutos de morangueiro 'San Andreas' foram colhidos em campo, em áreas de sistemas de produção convencional e orgânico, localizadas no município de Morro do Chapéu - BA, Brasil, situado nas coordenadas geográficas - $11^{\circ} 33^{\prime} \mathrm{S} 41^{\circ} 09^{\prime} \mathrm{O}$. O clima da região é tropical, classificado como Aw segundo a classificação de Köppen; Geiger (1928), com temperatura média anual de $20,2{ }^{\circ} \mathrm{C}$, e pluviosidade média anual de $691 \mathrm{~mm}$. O sistema de plantio dos morangueiros foi em solo com textura franco-argilosa, com sistema de irrigação por gotejo. 
No sistema orgânico de produção, os morangueiros apresentam certificação de origem por meio da Rede de Certificação Povos da Mata. No que se refere a produtividade, no sistema convencional foi de $800 \mathrm{~g} / \mathrm{planta} / \mathrm{ciclo}$, enquanto que no sistema orgânico foi de $1000 \mathrm{~g} / \mathrm{planta} /$ ciclo.

O cultivo do morango foi feito em solo, com canteiros cobertos com o filme plástico preto, de espessura mínima de 25 micras e largura de 1,60 m. Os canteiros foram sobreados com túneis baixos com altura de $0,8 \mathrm{~m}$, com distância entre arcos de 2,5 m, diâmetro do arco, em ferro galvanizado, de $5 \mathrm{~mm}$. plástico de 50 a 100 micra. As extremidades dos esteios eram acolchoadas com plásticos usados de cor branca, e a base do túnel teve em cada lado $10 \mathrm{~cm} \mathrm{a}$ mais do que a base do canteiro, e o filme plástico foi aberto na posição de descanso na altura de 30 a $40 \mathrm{~cm}$.

Os frutos foram colhidos frutos em estádio fenológico 9 (grau de maturação maduro) segundo Antunes et al. (2006), ou seja, com mais de $80 \%$ da superfície com a coloração vermelho-intensa (Figura 1), de forma manual. Os frutos foram transportados para o Laboratório de Armazenamento de Produtos Agrícolas (LAPA), da Universidade Federal do Vale do São Francisco campus Juazeiro/BA, sendo então selecionados de acordo com o estado fitossanitário, realizando-se o descarte dos frutos em estádio de senescência.

(A)

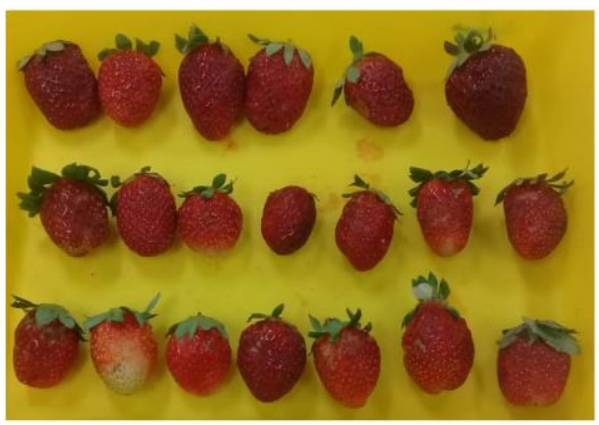

(B)

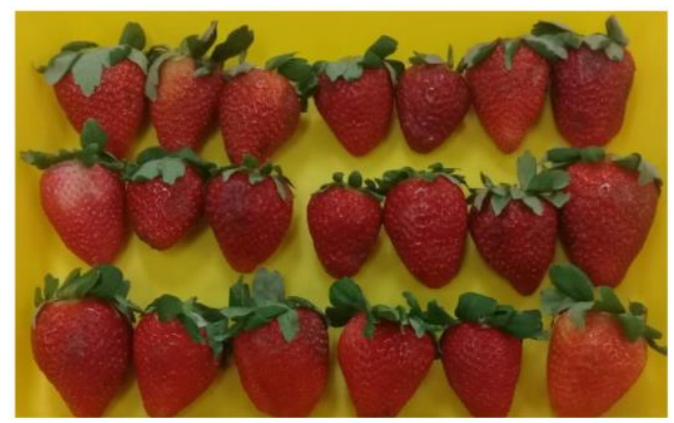

Figura 1. Morangos 'San Andreas' produzido em sistema convencional (A) e orgânico (B), em Morro do Chapéu - BA, em estádio de maturação maduro. 'San Andreas' strawberries produced in the conventional (A) and organic (B) system, at Morro do Chapéu - BA, at the ripe ripening stage.

Fonte: Autoria própria. Own authorship.

As avaliações físicas e físico-química foram realizadas em quatro repetições (quadruplicata), onde cada repetição foi composta por dez frutos. Para análise físico-química, os frutos foram destruídos e transformados em polpa em multiprocessador doméstico, sendo obtida uma amostra composta.

\section{Avaliações físicas dos morangos}

As análises físicas compreenderam: massa fresca do fruto, determinada por pesagem dos frutos individualmente em balança semi-analítica, com precisão de $0,01 \mathrm{~g}$; comprimento e largura dos frutos, determinado com o uso de paquímetro digital com precisão de $0,01 \mathrm{~mm}$; e firmeza da polpa, através da quantificação da força necessária para que uma sonda de $8 \mathrm{~mm}$, 
acoplada a um penetrômetro digital, modelo Instrutherm PTR-300, vencesse a resistência da polpa da fruta, sendo os resultados expressos em Newtons $(\mathrm{N})$.

\section{Avaliações físico-químicas do morango}

$\mathrm{O} \mathrm{pH}$ foi determinado através do método potenciométrico, com pHmetro digital de bancada, calibrado com soluções tampão $\mathrm{pH} 4,0$ e 7,0 a $20^{\circ} \mathrm{C}$ e precisão de 0,01 ; a acidez titulável expressa em ácido cítrico, foi determinada através de titulação ácido-base, utilizando solução de $\mathrm{NaOH}$ a 0,01 mol.L $\mathrm{L}^{-1}$ como agente titulante, e como indicador a solução alcóolica de fenolftaleína a 1\%; o teor de sólidos solúveis, foi determinado com uso do refratômetro digital marca Hanna, modelo Hi9601, com resultados expressos em ${ }^{\circ}$ Brix; o ratio foi calculado através da relação entre o teor de sólidos solúveis ( ${ }^{\circ}$ Brix) e acidez titulável expressa em ácido cítrico. Estas análises seguiram as metodologias descritas pelo Instituto Adolfo Lutz - IAL (2008).

O teor de ácido ascórbico (vitamina $C$ ) foi determinado por volumetria de óxidoredução empregando a solução de iodo a 0,015 mol. $\mathrm{L}^{-1}$ como agente titulante, e a solução de amido a 1\% como indicador, conforme metodologia descrita por Baccan et al. (2011).

\section{Análise de cor}

A cor do epicarpodos frutos foi avaliada utilizando um colorímetro digital portátil da marca Konica Minolta, modelo CR 10, com sistema de cor Cielab, sendo obtidos os parâmetros L, que indica luminosidade ou brilho e varia do claro (branco; 100) para o escuro (0:escuro/opaco); a, que indica a cromaticidade no eixo de cor verde(-) para vermelha (+); b, que indica a cromaticidade no eixo da cor azul (-) para amarela (+); $\mathrm{C}^{*}$ (cromaticidade ou intensidade da cor) e o ângulo Hue $\left({ }^{\circ} \mathrm{H}\right)$, onde $0^{\circ}=$ vermelho, $90^{\circ}=$ amarelo, $180^{\circ}=$ verde, $360^{\circ}=$ azul.

\section{Análise estatística}

Os dados experimentais foram submetidos ao teste de Shapiro-Wilk para verificar o grau de normalidade das variâncias. Os dados foram submetidos a análise de variância em delineamento inteiramente casualizado, e as médias comparadas por meio do teste t, ao nível de $5 \%$ de probabilidade $(\mathrm{p}<0,05)$. As análises estatísticas foram realizadas por meio do software SigmaPlot versão 12.0 .

\section{RESULTADOS E DISCUSSÃO}

De acordo com os resultados expressos na Tabela 1, pode-se afirmar que os morangos 'San Andreas' produzidos em sistema orgânico apresentaram maior tamanho.

Richter et al. (2018), encontraram valores próximos ao avaliarem os atributos físicos de morangos 'San Andreas' cultivados no município de Lages-SC em solo e hidroponia, encontraram valores médios para a massa fresca de 18,5 g, $34 \mathrm{~mm}$ para o comprimento, e 45 mm para a largura. 
Tabela 1. Avaliação física do morango 'San Andreas' produzido em sistema convencional e orgânico. Physical evaluation of 'San Andreas' strawberry produced in the conventional and organic system.

\begin{tabular}{lccc}
\hline Parâmetro & \multicolumn{2}{c}{ Sistema de cultivo } & P valor \\
\cline { 2 - 3 } & Convencional & Orgânico & \\
\hline Massa fresca $(\mathrm{g})$ & $12,40 \pm 2,99 \mathrm{~b}$ & $21,53 \pm 2,95 \mathrm{a}$ & 0,020 \\
Comprimento $(\mathrm{mm})$ & $35,89 \pm 3,33 \mathrm{~b}$ & $44,04 \pm 2,92 \mathrm{a}$ & 0,033 \\
Largura $(\mathrm{mm})$ & $26,60 \pm 2,55 \mathrm{~b}$ & $33,08 \pm 2,20 \mathrm{a}$ & 0,028 \\
Firmeza $(\mathrm{N})$ & $6,27 \pm 0,60 \mathrm{a}$ & $7,75 \pm 1,27 \mathrm{a}$ & 0,144 \\
\hline
\end{tabular}

As médias seguidas pela mesma letra não diferem estatisticamente entre si. Foi aplicado o teste t em nível de 5\% de probabilidade. P valor - nível descritivo ou probabilidade de significância. Means followed by the same letter, not differ from each other by the t test $(p<0.05)$. P-value - descriptive level or probability of significance.

Fonte: Autoria própria. Own authorship.

Em relação a firmeza da polpa dos frutos, não houve diferença significativa entre os sistemas de produção avaliados (Tabela 1). Resultado diferente ao relatado por Andrade et al. (2017), que ao avaliarem a qualidade de morangos 'Oso Grande' em sete pares de fazenda nos estados de São Paulo e Minas Gerais, Brasil, constataram que o sistema de cultivo orgânico proporcionou maior firmeza da polpa dos frutos em relação aos produzidos no sistema convencional.

Os morangos cultivados em diferentes sistemas de cultivo apresentaram valores de $\mathrm{pH}$ estatisticamente diferentes, com valores de 3,15 e 3,22 para o sistema de produção convencional e orgânico, respectivamente (Tabela 2). Tonin et al. (2017), ao avaliarem a qualidade de pseudofrutos de diferentes cultivares de morangueiro produzidos em sistema de produção orgânico em Cerro Largo- RS, encontraram valor de pH de 3,08 para a cultivar 'San Andreas', valor este inferior aos encontrados na presente pesquisa.

Tabela 2. Avaliação físico-química do morango 'San Andreas' produzido em sistema convencional e orgânico. Physical-chemical evaluation of 'San Andreas' strawberry produced in the conventional and organic system.

\begin{tabular}{lccc}
\hline Parâmetro & \multicolumn{2}{c}{ Sistema de cultivo } & \multirow{2}{*}{ P valor } \\
\cline { 2 - 3 } & Convencional & Orgânico & \\
\hline $\mathrm{pH}$ & $3,15 \pm 0,01 \mathrm{~b}$ & $3,22 \pm 0,02 \mathrm{a}$ & 0,002 \\
Acidez titulável (g de ácido cítrico.100g-1) & $0,334 \pm 0,006 \mathrm{a}$ & $0,259 \pm 0,005 \mathrm{~b}$ & $<0,001$ \\
Sólidos solúveis $\left({ }^{\circ} \mathrm{Brix}\right)$ & $7,27 \pm 0,06 \mathrm{a}$ & $7,1 \pm 0,01 \mathrm{~b}$ & 0,007 \\
Ratio & $21,36 \pm 0,286 \mathrm{~b}$ & $27,38 \pm 0,50 \mathrm{a}$ & $<0,001$ \\
Vitamina C (mg.100g $\left.{ }^{-1}\right)$ & $56,52 \pm 0,95 \mathrm{~b}$ & $76,89 \pm 0,96 \mathrm{a}$ & $<0,001$ \\
\hline
\end{tabular}

As médias seguidas pela mesma letra não diferem estatisticamente entre si. Foi aplicado o teste $t$ em nível de 5\% de probabilidade. P valor - nível descritivo ou probabilidade de significância. Means followed by the same letter, not differ from each other by the t test $(p<0.05)$. P-value - descriptive level or probability of significance.

Fonte: Autoria própria. Own authorship.

Em relação a acidez titulável expressa em ácido cítrico, os morangos produzidos em sistema convencional apresentaram menor acidez (Tabela 2). Os valores encontrados no 
presente trabalho foram inferiores aos encontrados em estudo desenvolvido por Cecatto et al. (2013), que comparou a produção de morangos de várias cultivares em solo e em substrato, em sistema convencional, em Passo Fundo - RS, encontrando valor médio de 1,00 g de ácido cítrico. $100 \mathrm{~g}^{-1}$ para o cultivar 'San Andreas'. Ainda segundo este autor, morangos de menor acidez são mais palatáveis e saborosos.

Em relação ao teor de sólidos solúveis ( ${ }^{\circ}$ Brix), houve diferença significativa entre os tratamentos (Tabela 2). Kobi et al. (2018), ao avaliarem o teor de sólidos solúveis de morangos 'Camarosa' e 'Albion' produzidos em cultivo orgânico e convencional no Planalto Central do Espírito Santo, não observaram diferença significativa deste parâmetro em função do sistema de cultivo. Tonin et al. (2017), ao avaliarem a qualidade de frutos de cultivares de morangueiro em sistema de produção orgânico, encontraram valor médio de 5,00 ${ }^{\circ}$ Brix, para a variedade em estudo.

O ratio traduz o grau de doçura e sabor do fruto constituindo um índice de qualidade referenciado, arremetendo a percepção do sabor "doce" e "ácido". De acordo com Cecatto et al. (2013), é desejável que frutos de morango apresentem teor mínimo de $7^{\circ} \mathrm{Brix}$, e valor máximo de acidez titulável em morangos para o consumo in natura é de 0,8 g de ácido cítrigo. $100 \mathrm{~g}^{-1}$. Portanto, evidencia-se que os sistemas de cultivo apresentam teores ideais para o consumo in natura, porém o cultivo em sistema orgânico proporcionou maior relação entre o teor de sólidos solúveis e acidez titulável (Tabela 2), evidenciando-se sabor mais doce e mais agradável.

Quanto ao teor de vitamina C, observou-se diferença significativa entre os morangos produzidos sob sistema convencional e orgânico (Tabela 2), uma vez que, os morangos produzidos em sistema orgânico apresentaram cerca de 36\% a mais. Musa et al. (2015), constataram o inverso, ao avaliarem o teor de Vitamina C em morangos 'San Andreas' de diferentes cultivares em sistemas de cultivo distintos no município de Bom Princípio - RS, com valores médios de 42,19 e $69,31 \mathrm{mg} 100 \mathrm{~g}^{-1}$ para morangos cultivados em sistema orgânico e convencional.

Tabela 3. Avaliação da cor do morango 'San Andreas' produzido em sistema convencional e orgânico. Evaluation of the color of the 'San Andreas' strawberry produced in a conventional and organic system.

\begin{tabular}{cccc}
\hline Parâmetro & \multicolumn{2}{c}{ Sistema de cultivo } & P valor \\
\cline { 2 - 3 } & Convencional & Orgânico & \\
\hline $\mathrm{L}^{*}$ & $36,34 \pm 2,90 \mathrm{a}$ & $37,29 \pm 0,89 \mathrm{a}$ & 0,614 \\
$\mathrm{a}^{*}$ & $33,01 \pm 0,89 \mathrm{a}$ & $34,77 \pm 1,19 \mathrm{a}$ & 0,111 \\
$\mathrm{~b}^{*}$ & $23,50 \pm 3,50 \mathrm{a}$ & $27,49 \pm 2,52 \mathrm{a}$ & 0,184 \\
Croma $^{\text {Ângulo Hue }}$ & $40,74 \pm 2,49 \mathrm{a}$ & $44,41 \pm 2,31 \mathrm{a}$ & 0,135 \\
& $35,05 \pm 3,87 \mathrm{a}$ & $38,08 \pm 2,02 \mathrm{a}$ & 0,296
\end{tabular}

As médias seguidas pela mesma letra não diferem estatisticamente entre si. Foi aplicado o teste t em nível de 5\% de probabilidade. $\mathrm{P}$ valor - nível descritivo ou probabilidade de significância. Means followed by the same letter, not differ from each other by the t test ( $p<0.05)$. P-value - descriptive level or probability of significance. Fonte: Autoria própria. Own authorship. 
No que concerne à coloração, constata-se que, não houve diferença significativa na cor do epicarpo dos frutos produzidos em diferentes sistemas de cultivo (Tabela 3).

Segundo Conti; Minami; Tavares (2002), valores menores que 29,24 indicam cor escura, enquanto valores de L* entre 29,34 e 34,62 indicam condição intermediária, e valores maiores que 34,62 cores clara. Diante disso, os autores devem afirmar os frutos avaliados neste estudo apresentaram coloração clara.Segundo Guedes et al. (2013), a coloração da fruta é de extrema importância para a aceitação inicial pelo consumidor, seguida da firmeza e do sabor.

Com relação aos valores de $\mathrm{a}^{*}$ e $\mathrm{b}^{*}$, de acordo com Machado et al. (2019), maiores valores de $a^{*}$ em relação ao valor de $b^{*}$, caracteriza um teor expressivo de compostos fenólicos como o licopeno, carotenoides e antocianinas, que consequentemente induz a uma maior cromaticidade dos frutos.

Analisando a coloração expressa em ângulo $\mathrm{Hue}^{\circ}$ que representa a tonalidade da cor da fruta, houve variações de 35,05 a 38,08 (Tabela 3), ou seja, indicando tonalidade vermelha. Esses valores encontrados para a coloração foram semelhantes aos encontrados por Franco et al. (2017), que ao analisarem a qualidade do morango 'San Andreas' submetido a diferentes posicionamentos de slab, densidades de plantio e meses de avaliação encontraram variações de 31,28 e 32,50 para o ângulo Hue, assumindo uma coloração vermelha.

\section{CONCLUSÃO}

Os morangos produzidos no sistema de produção orgânica em Morro do Chapéu - BA apresentaram maior massa fresca, comprimento e largura, resultando em frutos maiores.

Foi observado menores valores de acidez e sólidos solúveis, e maiores valores de $\mathrm{pH}$ e teor de vitamina $\mathrm{C}$ nos frutos produzidos em sistema de produção orgânica.

\section{AGRADECIMENTOS}

Ao Laboratório de Armazenamento de Produtos Agrícolas (LAPA) da Universidade Federal do Vale do São Francisco, junto a equipe, pelo auxílio e viabilização da realização do experimento.

\section{REFERÊNCIAS BIBLIOGRÁFICAS}

ANDRADE, C. A. W.; MIGUEL, A. C. A.; SPRICIGO, P. C.; DIAS, C. T. S.; JACOMINO, A. P. Comparison of quality between organic and conventional strawberries from multiple farms. Revista Brasileira de Fruticultura, Jaboticabal, v. 39, n. 2, p.1-9, 2017.

ANTUNES, L. E. C. Brasil é o maior produtor de morango da América do Sul. Campo \& Negócio - Hortifruti, Brasília, n. 7, p. 92-94, 2018.

BACCAN, N.; ANDRADE, J. C.; GODINHO, O. E. S; BARONE, J. S. Química analítica quantitativa elementar. São Paulo: Blucher, 2001. 324 p. 
CeCATto, A. P.; CAlvete, E. O.; NIENOW, A. A.; COSTA, R. C.; MENDONCA, H. F. C.; PAZINATO, A. C. Culture systems in the production and quality of strawberry cultivars. Acta Scientiarum Agronomy, Maringá, v. 35, n. 4, p. 471-478, 2013.

CONTI, J. H.; MINAMI K.; TAVARES, F, C. A. Produção e qualidade de frutos de morango em ensaios conduzidos em Atibaia e Piracicaba. Horticultura Brasileira, Brasília, v. 20, n. 1, p.10-17, 2002.

COSTA, A. F.; LEAL, N. R.; VENTURA, J. A.; GONÇALVES, L. S. A.; JÚNIOR, A. T. A.; COSTA, H. Adaptability and stability of strawberry cultivars using a mixed model. Acta Scientiarum Agronomy, Maringá, v. 37, n. 4, p.435-440, 2015.

DIAS, C. N; MARINHO, A. B.; ARRUDA, R. S.; SILVA, M. J. P. E.; DUARTE, E. P.; FERNANDES, C. N. V. Produtividade e qualidade do morangueiro sob dois ambientes e doses de biofertilizante. Revista Brasileira de Engenharia Agrícola e Ambiental, Campina Grande, v. 19, n. 10, p.961-966, 2015.

DIEL, M. I.; PINHEIRO, M. V. M.; THIESEN, L. A.; ALTISSIMO, B. S.; HOLZ, E.; SCHMIDT, D. Cultivation of strawberry in substrate: Productivity and fruit quality are affected by the cultivar origin and substrates. Ciência e Agrotecnologia, Lavras, v. 42, n. 3, p.229-239, 2018.

FAOSTAT. Food and Agriculture Organization of the United Nations Statistics Division. , 2019. Disponível em: http://faostat3.fao.org. Acesso em: 20 de nov. 2019.

FRANCO, E. O.; ULIANA, C.; LIMA, C. S. M. Características físicas e químicas de morango 'San Andreas' submetido a diferentes posicionamentos de slab, densidades de plantio e meses de avaliação. Revista Iberoamericana de Tecnologia Postcosecha, [s. l.], v. 18, n. 2, p.115-120, 2017.

GUEDES, M. N. S.; ABREU, C. M. P.; MARO L. A. C.; PIO R.; ABREU, J. R.; OLIVEIRA, J. O. Chemical characterization and mineral levels in the fruits of blackberry cultivars grown in a tropical climate at an elevation. Acta Scientiarum. Agronomy, Maringá, v. 35, n. 2, p.191-196, 2013.

INSTITUTO ADOLFO LUTZ - IAL. Métodos físico-químicos para análise de alimentos. São Paulo: IAL, 2008. 1020 p.

KOBI, H. B.; MARTINS, M. C.; SILVA, P. I.; SOUZA, J. L.; CARNEIRO, F.; HELENO, M. E. L. R.; QUEIROZ; N. M. B. COSTA. Organic and conventional strawberries: nutritional quality, antioxidant characteristics and pesticide residues. Fruits, Paris, v.73, n. 1, p.39-47, 2018.

MACHADO T. F.; MONTEIRO E. R.; TIECHER A. Estabilidade química, físico-química e antioxidante de polpa de Physalis pasteurizada e não pasteurizada sob congelamento. Brazilian Journal of Food and Technology, Campinas, v. 22, p.e2017149, 2019.

MUSA, C. I.; WEBER, B.; GONZATTI, H. C.; BIGUELINI, C. B.; SOUZA, G. C.; OLIVEIRA, E. C. Avaliação do teor de vitamina C em morangos de diferentes cultivares em sistemas de cultivo distintos no município De Bom Princípio/RS. Ciência e Natura, Santa Maria, v. 37, n. 2, p.368-373, 2015. 
REGANOLD, J. P.; WACHTER, J. M. Organic agriculture in the twenty-first century. Nature Plants, [s. 1.], v. 3, n. 2, p.e15221, 2016.

REGANOLD, J. P.; ANDREWS, P. K.; REEVE, J. R.; CARPENTER-BOGGS, L. SCHADT, C. W. Fruit and Soil Quality of Organic and Conventional. Plos One, [s. l.], v. 5, n. 10, p.e12346, 2010.

RICHTER, A. F.; FAGHERAZZI, A.F.; ZANIN, D. S.; CAMARGO, P.; ARRUDA, A. L.; KRETZCHMAR, A. A.; RUFATO, L.; SILVA, P. S. Produtividade e qualidade de cultivares de morangueiro sob cultivo de solo e semi-hidropônico. Revista Científica Rural, Santa Maria, v. 20, n. 1, p.193-203, 2018.

RUAN, J.; LEE, Y. H.; YEOUNG, Y. R. Flowring and Fruiting of Day-neutral and Everbearing Strawberry Cultivars in Highelevation for Summer and Autumn Fruit Production in Korea. Horticulture, Environment, and Biotechnology, [s. l.], v. 54, n. 2, p.109-120, 2013.

ŠAMEC, D.; MARETIĆ, M.; LUGARIĆ, I.; MEŠIĆ, A.; SALOPEK-SONDI, B.; Duralijab, B. Assessment of the differences in the physical, chemical and phytochemical properties of four strawberry cultivars using principal component analysis. Food Chemistry, London, v. 194, p.828-834, 2016.

SERVIÇO BRASILEIRO DE APOIO ÀS MICRO E PEQUENAS EMPRESAS - SEBRAE. Agronegócios: produção de morango. 2017. Brasília, DF, 2019. Disponível em: https://m.sebrae.com.br/Sebrae/Portal\%20Sebrae/UFs/BA/Anexos/Produ\%C3\%A7\%C3\%A3 o\%20de\%20morango\%20na\%20Bahia.pdf. Acesso em: 20 nov. 2019.

SHAW, D.; LARSON, K. D. Performance of early-generation and modern strawberry cultivars from the University of California breeding programme in growing systems simulating traditional and modern horticulture. Journal of Horticultural Science \& Biotechnology, Ashford, v. 83, n. 5, p.648-652, 2008.

TONIN, J.; MACHADO, J. T. M.; BENATI, J. A.; ROHRIG, B.; SOBUCKI, L.; CHASSOT, T.; SCHNEIDER, E. P. Yield and quality of fruits of strawberry cultivars in an organic production system. Científica, [s. l.], v.45, n. 3, p. 271-277, 2017.

UNITED STATES DEPARTMENT OF AGRICULTURE - USDA. Agricultural Research Service. National Nutrient Database for Standard Reference Legacy Release. Version Current: April. 2018. Disponível em https://ndb.nal.usda.gov/ndb/foods/show/09316. Acesso em: 20 nov. 2019.

XU, F.; SHI, L.; CHEN, W.; CAO, S.; SU, X.; YANG, Z. Effect of blue light treatment on fruit quality, antioxidant enzymes and radical-scavenging activity in strawberry fruit. Scientia Horticulturae, Amsterdam, v. 175, p.181-186, 2014. 